\title{
Cellular polypropylene electromechanical properties: exploring the nonlinear region
}

\author{
Pavlos Sgardelis ${ }^{1}$ (D) $\cdot$ Michele Pozzi $^{1}$
}

Received: 7 February 2018/ Accepted: 2 July 2018/Published online: 10 July 2018

(C) The Author(s) 2018

\begin{abstract}
Many studies have been conducted in the last decades on cellular polypropylene (Cell-PP) films. Most of them focus on the optimisation of the material for sensor applications. Processed under Gas Diffusion Expansion (GDE), Cell-PP films show high piezoelectric activity and low stiffness/density, properties that make them ideal for sensors. GDE increases the height and decreases the length over height ratio (aspect ratio) of individual voids within the material. This change in void morphology, and eventually stiffness, results in a nonlinear piezoelectric response of these materials. In this study, a CellPP sample was tested under static, quasi-static and low-frequency compressive stress. The main aim is to evaluate its mechanical and piezoelectric properties in the nonlinear region of its response over strain. The load-deflection curves as well as the piezoelectric responses were obtained for stresses up to $270 \mathrm{kPa}$ (engineering strain close to 0.26). It is shown that both the magnitude of the initial load and the strain rate have a critical effect on the creep/stress relaxation of the film and eventually on its piezoelectric response. Finally, it is shown that under dynamic conditions, and for the same engineering strain region, it is more relevant to present the piezoelectric response, in terms of strain rather than stress.
\end{abstract}

Keywords Cell-PP films $\cdot$ Polymers $\cdot$ High strain $\cdot$ Piezoelectric $\cdot d_{33} \cdot c_{33} \cdot$ Piezoelectrets $\cdot$ Ferroelectrets

\section{Introduction}

Film ferroelectrets, and among them cellular polypropylene (Cell-PP) developed in 1989 at Tampere University of Technology (TUT), gained interest in the last decades. Their mechanical properties (lightweight, mechanical flexibility, acoustic impedance close to that of air [1], endurance in high levels of strain) and piezoelectric properties (high $d_{33}$ constant up to $1400 \mathrm{pC} \mathrm{N}^{-1}$ under sub- $\mathrm{Hz}$ frequencies [2]), as well as their low cost, make them a good choice for a variety of applications.

Cell-PP films are created by bidirectional stretching of a polypropylene- $\mathrm{CaCO}_{3}$ particles mixture. By this procedure, voids of tens of microns thickness and hundreds of microns length are formed inside the bulk of the material.

Pavlos Sgardelis

p.sgardelis@ncl.ac.uk

Michele Pozzi

michele.pozzi@ncl.ac.uk

1 School of Engineering, Newcastle University, Claremont Road, Newcastle Upon Tyne, Tyne and Wear NE17RU, UK
These films can gain piezoelectric properties when charged under high fields. Both positive and negative polarity charges are trapped in either side of the voids surface. For this charge separation (discharge) to happen, the externally applied field should be large enough for a Townsend discharge to occur. The minimum field needed for a discharge event is called critical field $\left(E_{\mathrm{c}}\right)$ and is different for each void, depending on its height. The voids with the lowest critical field determine the critical field of the film (minimum field needed for the film to become electromechanically active). When the applied field is larger than this minimum, the charge density of the film (and as a result its piezoelectric coefficient $d_{33}$ ) increases linearly with the field [3] up to an equilibrium field where no more charges can be forced inside the material $[4,5]$. It has been shown that the equilibrium field is about two times the critical field of the film [4].

According to Paschen's law of critical breakdown field in micrometre voids, the thin voids of a virgin (non-treated) material have high $E_{\mathrm{c}}$. Therefore, the charge density of films with small voids is expected to be relatively small [6] compared to films with larger voids, assuming that both are 
charged under the same field. Large voids start to gain charge density at lower fields, and a higher piezoelectric coefficient $d_{33}$ is achieved by the end of the charging procedure [3, 6-8]. One method for actively increasing the thickness of the voids is the Gas Diffusion Expansion (GDE) procedure. During GDE, the pressure surrounding the sample is increased resulting in gas diffusion in the voids of the material after a long period of time (20-60 min). The pressure is then released quickly. The gas does not have the time to diffuse out of the voids, causing their expansion in the thickness direction, thereby reducing the length/height ratio (aspect ratio) [9, 10]. Tuncer [9] simulated the voided part of the material by a rhomboidal-like mesh structure and found that the aspect ratio of the voids is a critical parameter for the overall stiffness of the voided structure and as a result for the stiffness of the material in third direction $\left(c_{33}\right)$. However, neither the stiffness of the material nor the piezoelectric coefficient changes monotonically with the level of GDE [11]. Non-expanded and low expanded materials show a rapid increase of stiffness under compressive forces, as the voids collapse easily under small stresses due to their compliant structure (high aspect ratio). In this case, what is compressed is the bulk material rather than the air cavities, leading to high $c_{33}$ and low $d_{33}$. Expanding the film by GDE, and for moderate aspect ratios (still compliant voided structure), allows for higher strains to be applied, before the voids collapse, decreasing that way the $c_{33}$ and increasing the $d_{33}$. On the other hand, greater expansions lead to increment of $c_{33}$ as the voided structure becomes stiffer (low aspect ratio). Studies showed that the breakpoint that maximises the piezoelectric coefficient $d_{33}$ and lowers the stiffness $c_{33}$ is close to a density ratio of about 0.46 [12-14].

As compression of Cell-PP results in a similar but reverse effect to GDE procedure (decrease of void height and increase of aspect ratio), the above nonlinear effect of GDE on material stiffness implies that $d_{33}$ or/and $c_{33}$ are not constants during compression but depend on the applied stress over strain relationship that is also nonlinear. Under small compressive stress (no more than few $\mathrm{kPa}$ ), Cell-PP has a linear stress-strain response [15] (constant $c_{33}$ ) as well as constant piezoelectric coefficient $d_{33}[8,16]$. When the compressive stress is high enough (in the orders of tens to hundreds of $\mathrm{kPa}$ ), the piezoelectric response $[17,18]$, as well as the stiffness [19], does not depend linearly on stress. Kressmann [18] demonstrated that in the nonlinear region (up to $60 \mathrm{kPa}$ ), the stiffness of the Cell-PP sample initially decreases by almost $25 \%$ and then increases again. The opposite holds for $d_{33}$ versus stress.

The aim of this work is to study the direct and inverse piezoelectricity of Cell-PP films with focus in the nonlinear region (high stress and strain). Specifically, we explore the importance of the magnitude of the initial load and the strain rate in the creep/stress relaxation and the piezoelectric response of Cell-PP films.

\section{Materials and methods}

\section{Sample preparation}

The sample used is the EUH 75 Cell-PP film provided by Treofan Germany GmbH \& Co. KG. The film has nominal thickness of $75 \mu \mathrm{m}$ and external layers (one glossy and one mat) of solid polymer, enclosing the voided part of the material. The sample was placed in a high-pressure vessel and inflated with a GDE procedure, during which the pressure was increased to $5 \mathrm{MPa}$ and held for $60 \mathrm{~min}$. The pressure was then released from the vessel as quickly as possible, and the sample underwent a heat treatment for $10 \mathrm{~s}$ at $85{ }^{\circ} \mathrm{C}$ to make the inflation permanent.

The sample was charged in a custom-made negative corona triode, under constant current charging mode $[20,21]$. A square electrode of $12 \times 12 \mathrm{~mm}^{2}$ was made on the centre of the glossy side of the $15 \times 15 \mathrm{~mm}^{2}$ sample and then placed face down during charging. The nonelectrode area was left to avoid voltage tracking from the top surface to the bottom surface and eventually to the current $\left(I_{\mathrm{s}}\right)$-measuring probe, providing that way an equivalence to a guard ring commonly used [21]. Silver paint was used for creating the electrodes of the sample. Silver paint was preferred over other media, as it does not involve high temperatures while applying, avoiding the risk of thickness reduction of the sample [2].

A metal mesh (grid) exists between the corona tip, and the sample to ensure a uniform field is applied on the film. As only one power supply was available, the electric potential of the grid $V_{\mathrm{g}}$ was indirectly controlled by displacing the corona tip towards or away from the grid which was connected to a high voltage (HV) potential divider. By approximating the tip to the grid, more current is injected into it, which raises $V_{\mathrm{g}}$ as charges then need to flow to ground through the large resistance of the divider.

The corona tip was lowered until the current $I_{\mathrm{s}}$ flowing through the sample reached $0.02 \mu \mathrm{A}$. An uncertainty is involved in the measured current $I_{\mathrm{s}}$, as there is still a background current sensed continuously by the measuring tip. As the sample gets charged, the potential of its top surface rises. Its potential difference compared to that of the grid decreases, which drives $I_{\mathrm{s}}$ down. To keep the current $I_{\mathrm{s}}$ constant, the distance between the corona tip and the grid is controlled in close loop, leading to the potential difference between the grid and the sample constant. The field below the grid builds up to a point where it gets larger than the dielectric strength of the material and a breakdown 
across the sample occurs. This is indicated by a rapid increment of $I_{\mathrm{s}}$, without any increment of $V_{\mathrm{g}}$ by the displacement of the corona tip. To avoid the discharge, the corona was manually switched off just before this event. After charging, the sample was cut down to the $12 \times 12 \mathrm{~mm}^{2}$ electrode area and the second electrode was applied on the other surface. Cross-sectional images of the sample were taken with the Scanning Electron Microscope (SEM) Hitachi TM3030 for estimation of the sample total thickness, as well as the thickness of the external solid layers. Both quantities were measured after the charging procedure to account for thickness changes following the process. Ten cross-sectional images were analysed by a custom-made image processing software for the above measurement.

The sample was sandwiched between two metal plates of the same area as the sample $\left(12 \times 12 \mathrm{~mm}^{2}\right)$. In order to provide a uniform pressure to the whole area of the sample, as well as a better conductive continuity, two media were inserted and tested in between the sample and the metal plates: conductive silicone pads and an extra thin layer of silver paint. The sample was tested with and without these media for its static piezoelectric activity.

\section{Static, quasi-static tests}

After the best medium was chosen, the sample was inserted into a custom-made rig that permits simultaneous compression and application of voltage to the film, for the inverse piezoelectric effect to be tested. The sample was tested under a variety of static stresses and voltages in both the linear and the nonlinear region. The strain was measured with the Microepsilon Capa NCDT 6200 capacitive displacement sensor module, using the CS05 sensor.

Static step compression tests were performed to obtain the quasi-static creep response under various loads ranging from 4 to $270 \mathrm{kPa}$. The load was statically applied and held while the charges and strain produced were logged with a Keithley $6517 \mathrm{~b}$ electrometer and the capacitive displacement sensor, respectively.

\section{Dynamic tests}

Stress relaxation and creep tests were carried out, using a Shimadzu EZ-SX under a range of strain ramps while charges, strain and the stress were simultaneously logged. At the end of each ramp, the material was left to relax and the creep/stress relaxation was observed. In addition, different stress rates were applied to the film in order to investigate the effect of the loading rate on both the charges and the strain.

\section{Results}

The current $I_{\mathrm{s}}$ flowing through the sample during the negative constant current corona charge is shown in Fig. 1. The end of the charging process was designated with the spontaneous rise of $I_{\mathrm{s}}$ to a maximum of $0.048 \mu \mathrm{A}$ after $70 \mathrm{~s}$ of charging. At that point, the corona device was manually switched off. The rapid increment in current is attributed to the material's dielectric strength being reached.

From the ten cross-sectional images of the film analysed, the average thickness of the sample was estimated to be $107 \pm 3 \mu \mathrm{m}$ including $10 \mu \mathrm{m}$ of the external solid layers. The uncertainty is mostly due to the irregular shape of the film (Fig. 2). The external layers are made of solid polypropylene and are almost three orders of magnitude stiffer than the total stiffness of the Cell-PP material (1.5-2 GPa compared to $1-4 \mathrm{MPa}$, respectively). It is assumed that only the voided part of the sample gets compressed, so the active thickness of the sample is $97 \pm 3 \mu \mathrm{m}$.

The addition of either conductive silicone pads or extra silver paint between the samples and the metal plates was found to increase the piezoelectric response obtained, compared to the case where no medium was used (Fig. 3). In the absence of media, the obtained piezoelectric activity was lower by more than $35 \%$. This is thought to be due to an unsatisfactory mechanical and electrical contact in their absence. The conductive silicone pads have nominal thickness of $500 \mu \mathrm{m}$ and measured compressive stiffness of 1.5 MPa. As these pads are five times thicker than the CellPP and have comparable stiffness, large uncertainties are introduced in measuring the strain of the sample, making it

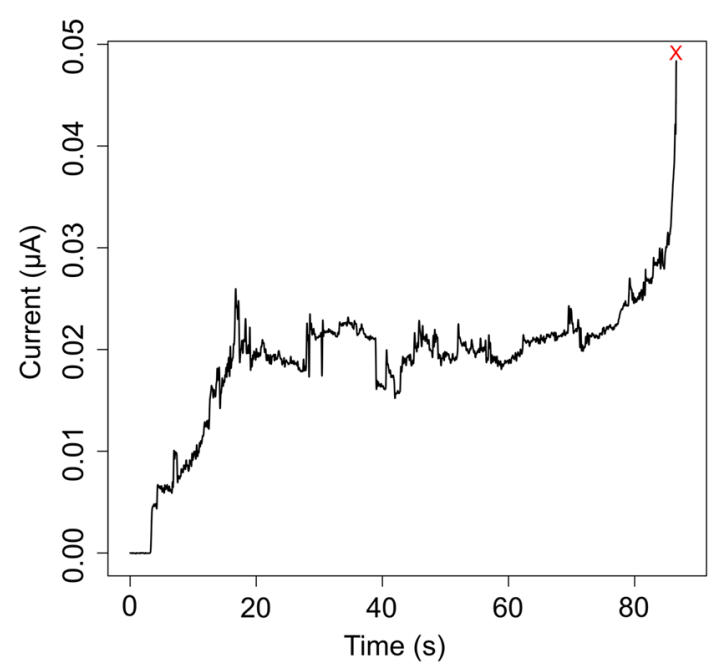

Fig. 1 Current $I_{\mathrm{s}}$ flowing through the sample during negative constant current corona charge. Grid to sample distance was $d_{\mathrm{g}}=5 \mathrm{~mm}$ and corona voltage $V_{\mathrm{c}}=25 \mathrm{kV}$. The "X" mark indicates the time the corona tip voltage was switched off 


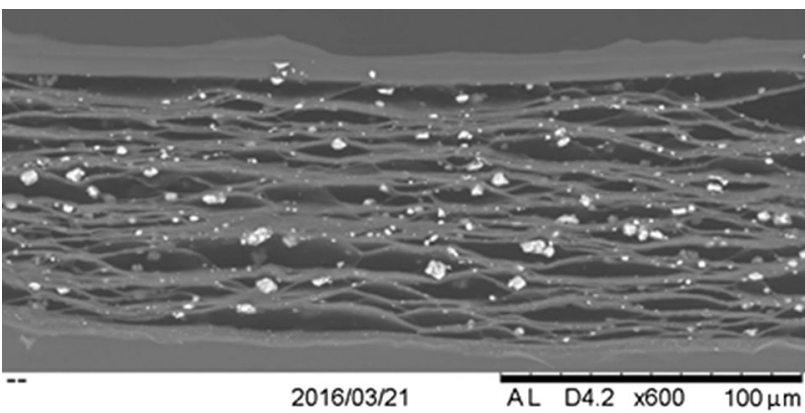

Fig. 2 SEM cross-sectional image of the sample

a non-valid choice for strain measurements. The extra layer of silver paint was eventually chosen for the rest of the experiments.

There is a slight increment in the slope of the charge production after $20 \mathrm{kPa}$ (Fig. 3a), indicating the nonlinearity of the response. This is better shown in Fig. 3b where the piezoelectric coefficient $d_{33}$ is plotted over stress.

In terms of strain, the linear region occurs under small displacements, so it can either be tested by the use of small stresses, or by the inverse piezoelectric effect that induces small strains. The inverse piezoelectric response of the material was tested under low fields. The obtained strain and piezoelectric coefficient are presented over a variety of step excitation voltages up to $200 \mathrm{~V}$ in Fig. $4 \mathrm{a}$, b, respectively. The engineering strain instead of the true strain is given for this test, as we are interested in the change of thickness compared to the initial thickness of the film.

The highest strain obtained by the application of the external field is rather small (Fig. 4a), indicating that the film is still within the linear region of operation. The piezoelectric coefficient (Fig. 4b) seems to increase slightly over the applied potential range. This increase, however, is not significant due to the high deviation; thus, the response is considered to be constant (around $130 \mathrm{pm} \mathrm{V}^{-1}$ ). To test the inverse piezoelectric effect in the nonlinear region, the sample was preloaded under various static compressive stresses to induce large strains. Without these preloads, the electric field needed to obtain higher strains would be high enough to simply recharge the film.

The final strain obtained at the end of the creep period was measured after the application of static stress. The results are shown in Fig. 5.

Figure 6 shows the static inverse piezoelectric response of the film under a variety of static stresses.

It can be inferred that higher $d_{33}$ can be achieved when working under higher strains. The sample was tested for its quasi-static response under various static compressive loads. The sample's engineering strain, the stress and the produced charges, given as a function of time, are presented in Fig. 7. For clarity, only a selection of the stresses applied is shown.

As shown in Fig. 7, each curve obtained is constituted of two main regions. The first region starts at $t=0$, when the static load is applied, and finishes when the stress reaches its maximum. As the stress is applied in a short ramp, this first region is very brief, lasting from 0.2 to $1 \mathrm{~s}$ for higher stresses. After $t=1 \mathrm{~s}$, stress is constant and a creep-like response is observed in the charges and the strain that can last for 1-150 s depending on the static stress applied.

From the data shown in Fig. 7, it is evident that charges are being produced during creep (after the stress reaches its maximum). The relationship between charges produced and strain is not linear. For example, as the $20 \mathrm{kPa}$ load is applied, the strain is close to 0.05 and the obtained charges are $0.5 \mathrm{nC}$. By comparing with the charges produced under $30 \mathrm{kPa}$ ( $0.8 \mathrm{nC}$ for 0.06 strain), it can be inferred that more charges were produced per unit of strain for higher stresses.

Further investigation of this nonlinearity is done by applying stress ramps to the sample, while logging the charges and the strain. Figure 8a, b shows the charges plotted against the engineering strain and stress, respectively.
Fig. 3 a Charges and b piezoelectric coefficient $d_{33}$ over static stress for different media applied between the sample and the metal plates. Each data point represents the average of five measurements. Before the application of each new stress level, the film was left to relax back to its initial thickness. All data were obtained with the same sample
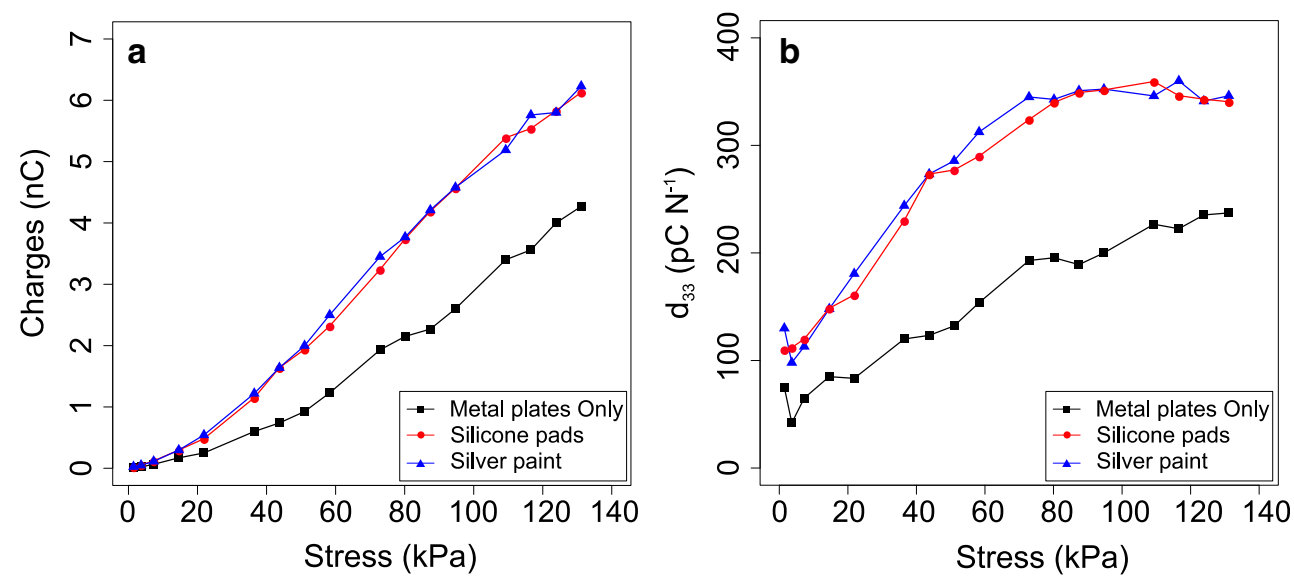
Fig. 4 Strain a and inverse piezoelectric effect b over applied electric potential. A stress of $1.16 \mathrm{kPa}$ acts as a preload to ensure reliable electrical and mechanical contacts. The result is an average of three runs
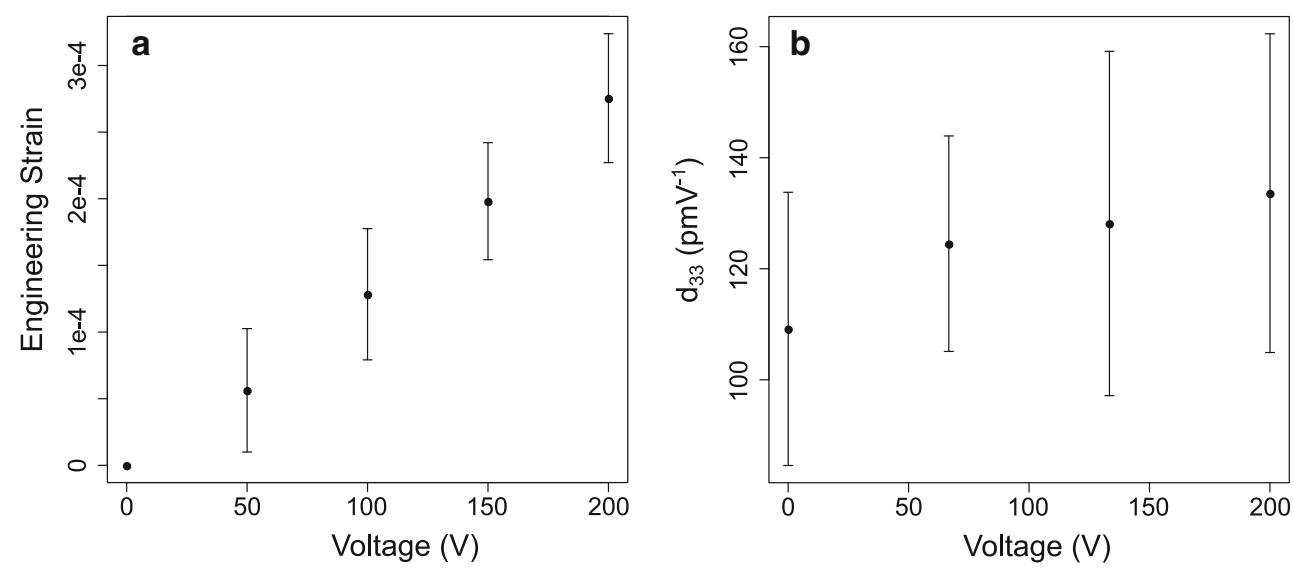

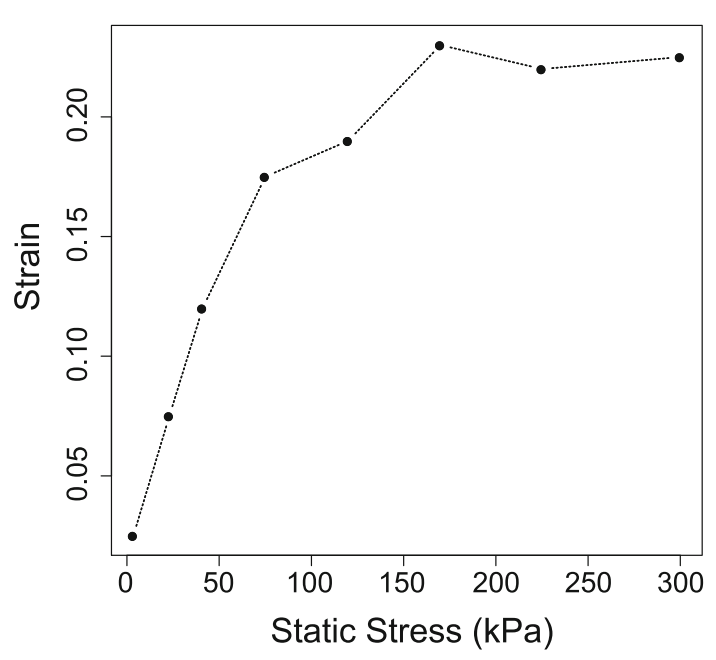

Fig. 5 Strain obtained for various static stresses applied. The line is a guide to the eye. Single-run results

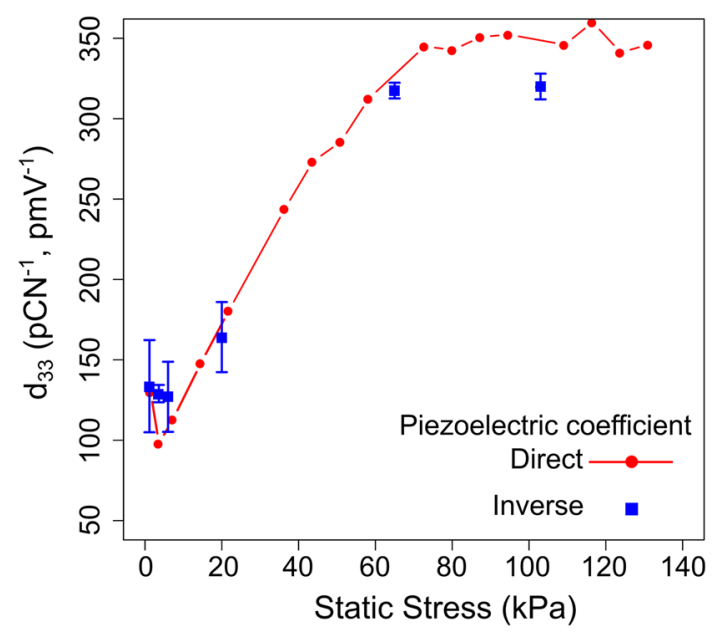

Fig. 6 Comparison between the direct static $d_{33}$ with data taken from Fig. 3 and inverse piezoelectric response of the film compressed under various static stresses. The result is the average of five runs

This test is neither stress nor strain controlled. The controlled parameter is the displacement of the head of the

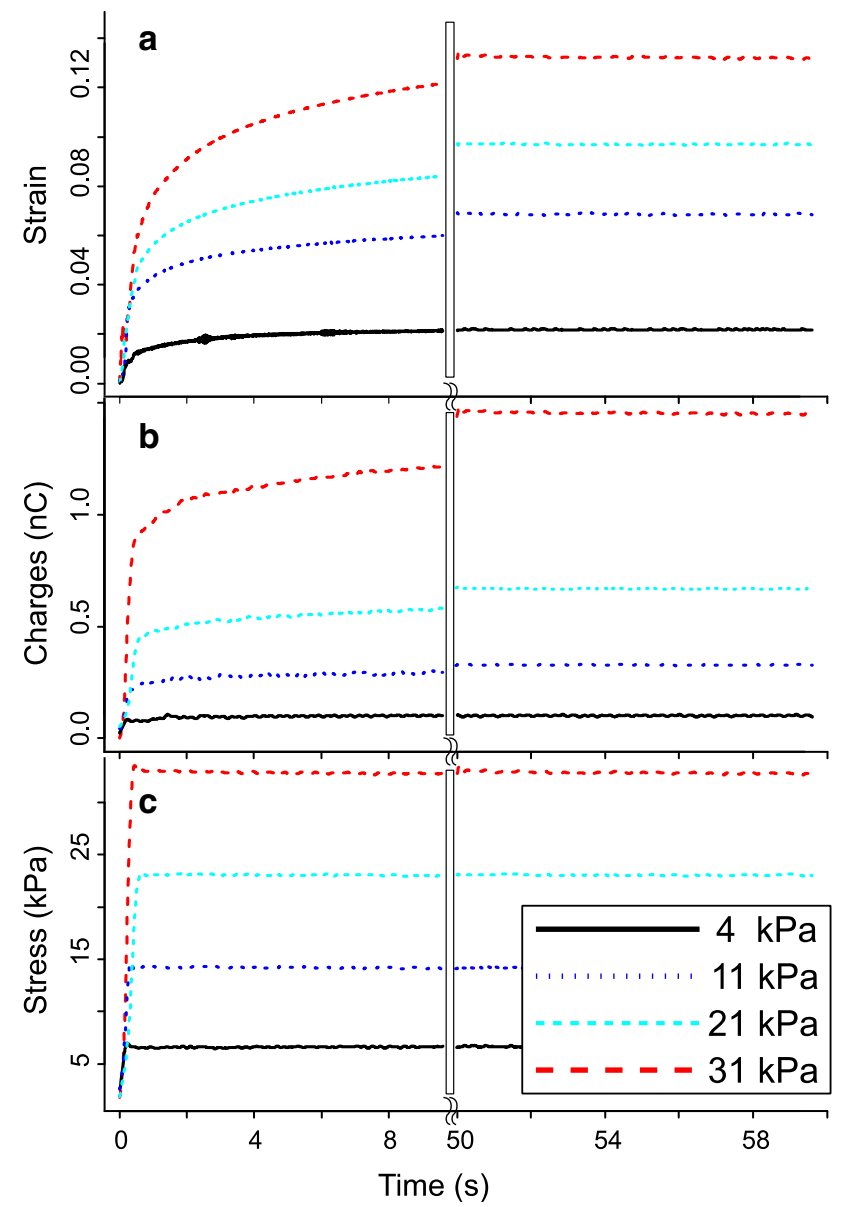

Fig. 7 Quasi-static response of the sample to various compressive loads. The figure shows the compressive strain a and the charges b that the stress $\mathbf{c}$ produced. Single-run results plotted. The preload is $3 \mathrm{kPa}$

machine, which is done in a linear manner. By the end of the displacement ramp, the head of the machine is stopped, leading to the observed stress relaxation and creep response. The further displacement observed in this later stage is possible due to elasticity of the machine's loadbearing components. As Fig. 8 suggests, charges are being 
Fig. 8 a Charges over engineering strain and $\mathbf{b}$ charges over stress, for maximum engineering strains of $0.04,0.07$ 0.15 and 0.18 . Graph shows stress relaxation response at the end of each ramp. The result is an average of two runs. The preload for this test is $6 \mathrm{kPa}$

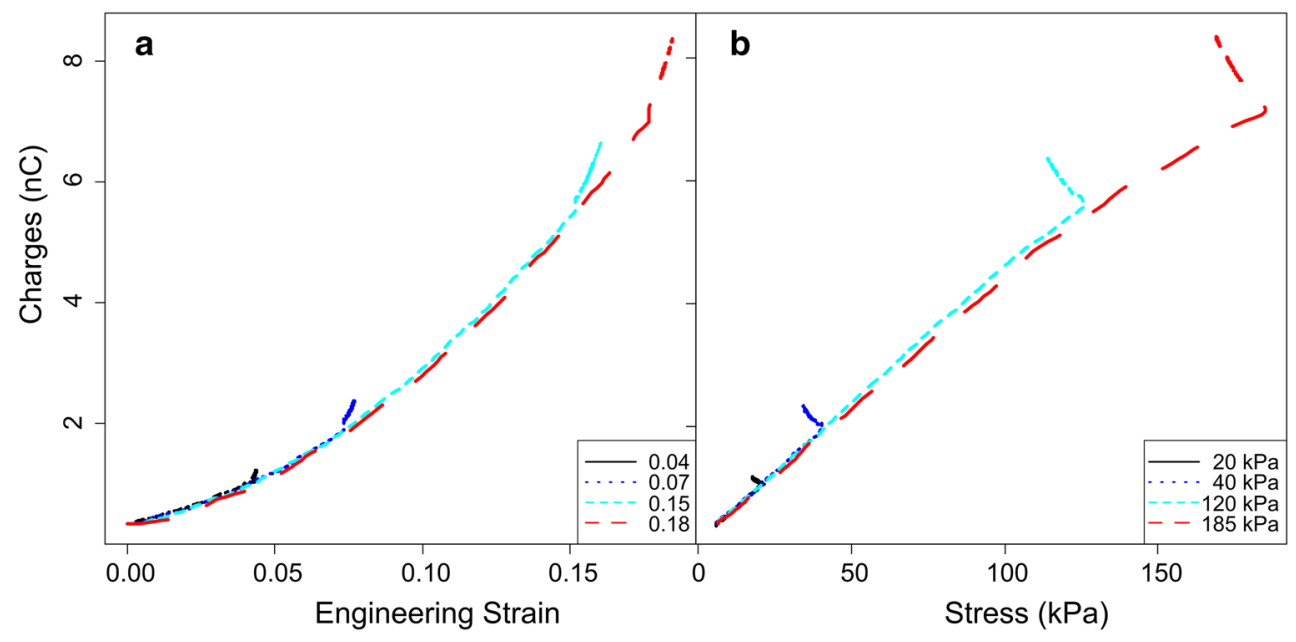

produced in accordance with the creep/stress relaxation. At the end of each ramp, charges are still positively correlated with the strain (albeit at a different rate), whereas they are inversely correlated with the stress. The different rate at which the charges are produced during relaxation implies that there is also correlation between the stress rate and the charge production rate. To test this, different stress rates were applied to the film and the charges were logged. Figure 9 clearly demonstrates that the charges being produced are related to the stress slope.

Less charges and lower strain are observed for higher stress rates. The final strain obtained for the 150 and 300 $\mathrm{kPa} \mathrm{s}^{-1}$ ramps is almost the same and comparable to the final strain obtained with the $300 \mathrm{kPa}$ step response in Fig. 5. Note that the functional form of the response over engineering strain is concave while that over stress is convex.

\section{Discussion}

The piezoelectric response of Cell-PP films was tested under static, quasi-static and low-frequency compressive stress. Before applying the stress, the film was treated with Gas Diffusion Expansion and charged with a custom-devised corona charger. An extra thin layer of silver paint was applied between the sample and the metal plates to provide a gap-free media and better load distribution (Fig. 3). Such a continuous medium is required, in order to extract the total amount of charges produced on the external layers of the polymer during compression. Silver epoxy has been reported to reduce the measured piezoelectric properties as well as the dielectric properties of a PZT-polymer material by 25 and $40 \%$, respectively [22], compared to evaporated silver electrodes. This is attributed to air gaps existing between the epoxy and the surface of the material. Similar results are presented here as by only

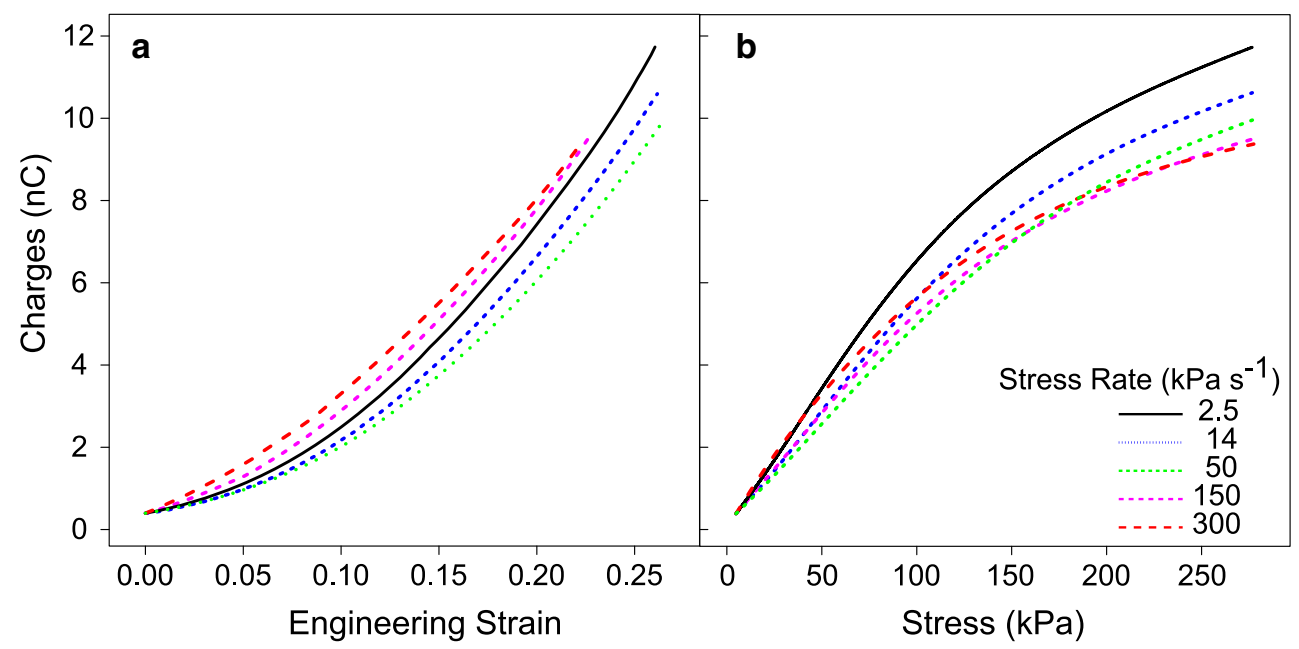

Fig. 9 a Charges produced over engineering strain and $\mathbf{b}$ charges produced over stress, for different stress rates. The final stress for every curve is $270 \mathrm{kPa}$. The result is an average of two runs 
having the silver electrodes the piezoelectric response is decreased. It is shown, in our case, that an extra layer of silver paint between the metal plates and the electrodes of the sample provides a satisfactory gap-free media for obtaining the total response of the film.

Morphologically different voids attained different charge densities by the end of the charging procedure and produce charges under different stress levels, as their thickness and stiffness vary. As there is a broad distribution of dissimilar voids in the film, the total charge produced (and the resulting $d_{33}$ ) varies in accordance with the compressive stress applied on the film. This yields a smooth response over the range of stress which, however, is not linear. Under low stress, the film's piezoelectric coefficient and stiffness are constant, giving linear strain over voltage response (Fig. 4a). The range of strain over which the response is linear depends on the morphology of the material's inner structure. On the contrary, under higher stress, the produced strain and charges are not linearly dependent on stress. This is an outcome of mostly the larger, less stiff, voids being engaged under low stress and smaller voids being engaged only under increasing stress, contributing to a rather smooth increase of the charges produced, with a rate of increase depending on the abundance of voids with a given thickness, their charge density and their stiffness. Evidence that void geometry is an important determinant of the piezoelectric behaviour of polymer films comes from experiments with laboratorymade films with voids of the same morphology. These laboratory-made films usually have large voids, which are quickly engaged at low stress levels leading to sharp drop of piezoelectric activity when higher stress levels are applied [23].

It is known that Cell-PP becomes stiffer (higher $c_{33}$ ) in the nonlinear region of high strains. Due to the mechanism of charge production, discussed, for example, by Dreyfus and Lewiner [24], there is a strong correlation between the strain and the charges (Fig. 8a). As a result, the strain is more directly correlated with the charges produced than the stress (Fig. 8). Whereas under low compressive stress, in the linear region, both strain and stress are valid choices as state variables, at higher compressive stress, it becomes mandatory to use strain.

Based on the above, one needs to define the stress, or equilibrium strain (after creep), region of operation when measuring quantities like stiffness $\left(c_{33}\right)$, piezoelectric coefficient $\left(d_{33}\right)$, electromechanical coupling coefficient $k^{2}$, etc. Furthermore, the direct piezoelectric coefficient is expressed as charges produced per unit force, while the inverse piezoelectric effect is expressed as displacement per unit voltage. For those two values to be comparable, as observed in Fig. 6, it is important that they are investigated under similar strain. In addition, when under quasi-static
(Fig. 7) or dynamic compression (Figs. 8, 9), where creep or stress relaxation is involved, it is obvious that there is a strong relationship between strain and the charges obtained.

As creep/stress relaxation takes place during compression, it is not surprising that the stress rate influences the strain rate and the rate of charges produced (Fig. 9). For higher stress rates, creep does not have the time to develop; thus, less displacement is observed. As a result, fewer charges are produced under the same target stress. This phenomenon can be clearly seen on the higher charges produced over strain for the creep response (stress rate $=0$ ) compared to the charges produced during the ramp (stress rate $\neq 0$ ) (Fig. 8).

The final strain obtained increases moderately with the stress rate (Fig. 9a, 2.5-50 $\mathrm{kPa} \mathrm{s}^{-1}$ ) for low stress rates. However, further increment of the stress rate (150 and 300 $\mathrm{kPa} \mathrm{s}^{-1}$ ) results in a marked decrease in the final strain. This change of behaviour can be attributed to the increment of storage modulus of the material, when under high dynamic excitation [15]. The general effect of stress rate is that more charges are produced at low rates. Whereas the charge production rate consistently increases with the strain state (concave curves in Fig. 9a), when considered as a function of stress, a steeper start at low stress values is followed by a progressively reduced growth (convex curves in Fig. 9b). The interpretation is that further deformation of the material makes it more electrically active by engaging more voids, albeit, at the same time, the increased stiffness reduces the yield as a function of stress.

\section{Conclusions}

Functionalised Cell-PP exhibits strong nonlinearities. It is important to consider and optimise the material for the region of strain that it will operate in via GDE. As a variety of applications exists, differently prepared films will be suitable, according to the available surface area, strain levels, forces present and their rate of change. As the piezoelectric coefficient $d_{33}$ more than doubles in the nonlinear region before it decreases again, the associated nonlinearity might be beneficial for applications that need high piezoelectric coefficient $\left(d_{33}\right)$ as well as stiffness $\left(c_{33}\right)$.

When under static mode, the piezoelectric parameters can be expressed either as a function of the applied static stress or the final strain (total strain after creep). On the contrary, during quasi-static and dynamic response, there is creep/stress relaxation taking place and the time dimension of the response must be suitably included.

Acknowledgements The authors would like to thank Treofan Germany $\mathrm{GmbH} \& \mathrm{Co}$. KG, for providing the cellular polypropylene 
samples. PS acknowledges the financial support (studentship) he received from the School of Mechanical and Systems Engineering, Newcastle University, UK. Comments by two unknown reviewers of this journal on a previous version of this work are highly acknowledged.

\section{Compliance with ethical standards}

Conflict of interest There are no conflicts of interest to the best of our knowledge.

Open Access This article is distributed under the terms of the Creative Commons Attribution 4.0 International License (http://creative commons.org/licenses/by/4.0/), which permits unrestricted use, distribution, and reproduction in any medium, provided you give appropriate credit to the original author(s) and the source, provide a link to the Creative Commons license, and indicate if changes were made.

\section{References}

1. Sborikas, M., Wegener, M.: Cellular-foam polypropylene ferroelectrets with increased film thickness and reduced resonance frequency. Appl. Phys. Lett. 103(25), 252901 (2013)

2. Zhang, X., Hillenbrand, J., Sessler, M.: Improvement of piezoelectric activity of cellular polymers using a double-expansion process. J. Phys. D Appl. Phys. 37, 2146-2150 (2004)

3. Mellinger, A., Mellinger, O.: Breakdown threshold of dielectric barrier discharges in ferroelectrets: where Paschen's law fails. IEEE Trans. Dielectr. Electr. Insul. 18(1), 43-48 (2011)

4. Zhang, P., Xia, Z., Qiu, X., Wang, F., Wu, X.: Influence of charging parameters on piezoelectricity for cellular polypropylene film electrets. In: IEEE International Symposium on Electrets, pp. 39-42 (2005)

5. Altafim, R.A.C., Altafim, R.A.P., Basso, H.C., Quin, X., Wirges, W., Gerhard, R., Jenninger, W., Wagner, J.: Dielectric barrier discharges in multi-layer polymer ferroelectrets. In: IEEE Conference on Electrical Insulation and Dielectric Phenomena, pp. 142-145 (2009)

6. Harris, S., Mellinger, A.: Pressure dependence of space charge deposition in piezoelectric polymer foams: simulations and experimental verification. Appl. Phys. A 107(3), 553-558 (2012)

7. Zhang, X., Hillenbrand, J., Sessler, G.M.: Piezoelectric $d_{33}$ coefficient of cellular polypropylene subjected to expansion by pressure treatment. Appl. Phys. Lett. 85(7), 1226-1228 (2004)

8. Hillenbrand, J., Zhang, X., Zhang, Y., Sessler, G.M.: Pressuretreated cellular polypropylene with large piezoelectric coefficients. In: IEEE Annual Report Conference on Electrical Insulation and Dielectric Phenomena, pp. 40-43 (2003)

9. Tuncer, E.: Numerical calculations of effective elastic properties of two cellular structures. J. Phys. D Appl. Phys. 38(3), 497-503 (2005)

10. Sgardelis, P., Pozzi, M.: An FE model of a cellular polypropylene: exploring mechanical properties. Proc. SPIE Microtechnol. 10246, 11 (2017)
11. Hillenbrand, J., Sessler, G., Zhang, X.: Verification of a model for the piezoelectric $d_{33}$ coefficient of cellular electret films. J. Appl. Phys. 98(6), 064105 (2005)

12. Wegener, M., Wirges, W., Fohlmeister, J., Tiersch, B., GerhardMulhaupt, R.: Two-step inflation of cellular polypropylene films: void-thickness increase and enhanced electromechanical properties. J. Phys. D Appl. Phys. 37(4), 623-627 (2004)

13. Wegener, M., Tuncer, E., Gerhard-Mulhaupt, R., Bauer, S.: Elastic properties and electromechanical coupling factor of inflated polypropylene ferroelectrets. In: IEEE Conference on Electrical Insulation and Dielectric Phenomena, pp. 752-755 (2006)

14. Wegener, M., Wirges, W., Gerhard-Multhaupt, R., Dansachmüller, M., Schwödiauer, R., Bauer-Gogonea, S., Bauer, S., Paajanen, M., Minkkinen, H., Raukola, J.: Controlled inflation of voids in cellular polymer ferroelectrets: optimizing electromechanical transducer properties. Appl. Phys. Lett. 84(3), 392-394 (2004)

15. Gaal, M., Bovtun, V., Stark, W., Erhard, A., Yakymenko, Y., Kreutzbruck, M.: Viscoelastic properties of cellular polypropylene ferroelectrets. J. Appl. Phys. 119(12), 125101 (2016)

16. Daosheng, P., Xiaoqing, Z., Xuewen, W., Gongxun, C., Shuo, Z., Hongqiang, L., Zhongfu, X.: Piezoelectricity of cross-linked polypropylene films treated by hot-stretching. In: IEEE 9th International Conference on the Properties and Applications of Dielectric Materials, pp. 1166-1169 (2009)

17. Hillenbrand, J., Xia, Z., Zhang, X., Sessler, G.M.: Piezoelectricity of cellular and porous polymer electrets. In: Proceedings. 11th International Symposium on Electrets, pp. 46-49 (2002)

18. Kressmann, R.: Linear and nonlinear piezoelectric response of charged cellular polypropylene. J. Appl. Phys. 90(7), 3489-3496 (2001)

19. Cronin, D.S., Ouellet, S.: Low density polyethylene, expanded polystyrene and expanded polypropylene: strain rate and size effects on mechanical properties. Polym. Test. 53, 40-50 (2016)

20. Giacometti, J., Fedosov, S., Costa, M.: Corona charging of polymers: recent advances on constant current charging. Braz. J. Phys. 29(2), 269-279 (1999)

21. Giacometti, J., Campos, S.: Constant current corona triode with grid voltage control. Application to polymer foil charging. Rev. Sci. Instrum. 61(3), 1143-1150 (1990)

22. Sherrit, S., Gauthier, N., Wiederick, H.D., Mukherjee, B.K., Prasad, S.E.: The effect of electrode materials on measured piezoelectric properties of ceramics and ceramic-polymer composites. In: [Proceedings] 1990 IEEE 7th International Symposium on Applications of Ferroelectrics, pp. 346-349 (1990)

23. Pisani Altafim, R., Rychkov, D., Wirges, W., Gerhard, R., Basso, H., Correa Altafim, R., Melzer, M.: Laminated tubular-channel ferroelectret systems from low-density polyethylene films and from fluoroethylene- propylene copolymer films - a comparison. IEEE Trans. Dielectr. Electr. Insul. 19(4), 1116-1123 (2012)

24. Dreyfus, G., Lewiner, J.: Free energy of electrets. Phys. Rev. B 14(12), 5451-5457 (1976)

Publisher's Note Springer Nature remains neutral with regard to jurisdictional claims in published maps and institutional affiliations. 